\title{
Le regard suédois sur les femmes écrivains de la francophonie
}

Ylva Lindberg

Université de Jönköping

\section{L'Afrique au féminin}

Cette étude est consacrée aux femmes écrivains désignées en Suède comme issues de la francophonie subsaharienne. Le choix de cette écriture féminine est doublement intéressante, car les femmes ont dû se battre autant contre les normes patriarcales leur interdisant de prendre la parole par la plume, que contre les normes raciales interdisant aux noirs de s'élever au niveau intellectuel des blancs (Coly, 20I0 : I25). À cette lutte entre les races s'ajoute l'histoire de l'esclavage, sans commune mesure avec les abus d'autres peuples dans le monde. À en croire Stefan Helgesson, qui a mené plusieurs projets sur la world literature ${ }^{\mathrm{I}}$ sous les auspices du post-colonialisme ${ }^{2}$, l'écriture féminine du continent africain noir s'est lentement frayée un chemin vers le public à partir des années I980 jusqu'à nos jours. Selon lui, ce n'est qu'autour de 2005 que l'histoire du post-colonialisme racontée par les femmes en Afrique noire a véritablement été accessible en suédois (Helgesson, 2007). Sans prétendre à ce que toute littérature féminine et francophone de l'Afrique sub-saharienne porte des empreintes postcoloniales, nous allons voir que cette perspective influe sur la réception suédoise, telle qu'elle se présente dans la presse journalistique et dans les revues. En étudiant comment les critiques en Suède commentent et interprètent les œuvres et les auteurs de notre corpus, nous espérons mettre en lumière les pratiques de labellisation dans la diffusion mondiale des lettres.

Nous avons restreint le corpus aux auteures nées après I960. Ces générations sont ancrées dans la diversité culturelle et linguistique du $\mathrm{XXI}^{\mathrm{e}}$ siècle et font montre d'approches distancées et personnalisées de l'histoire de l'indépendance de l'Afrique noire. Par conséquent, leur

How to cite this book chapter:

Lindberg, Y. 20I 5. Le regard suédois sur les femmes écrivains de la francophonie. In: Cedergren, M. et Briens, S. (eds.) Médiations interculturelles entre la France et la Suède. Trajectoires et circulations de I945 à nos jours. Pp. 44-55. Stockholm: Stockholm University Press. DOI: http://dx.doi.org/IO.I6993/bad.e. License: CC-BY 
littérature n'est ni au service du colonisé ni du colonisant, pas plus qu'elle ne sert nécessairement d' " arme contre le néocolonialisme ou l'impérialisme français»(Coste, 20I0). Sans apparentes similitudes entre elles, ces auteures sont plutôt les disciples de Maryse Condé, laquelle refusa de se laisser enfermer dans l'exaltation d' " un monde noir » (ibid.).

\section{La cartographie de Världslitteratur.se}

Ces dernières années en Suède se démarquent par un intérêt croissant pour la world literature (Le Bris \& Rouaud, 2007), c'est-à-dire pour une littérature issue d'autres horizons que ceux de l'Europe et des ÉtatsUnis (Lindberg, 20IO). En 20I2, le site Världslitteratur.se est lancé afin de mettre en avant la littérature mondiale traduite en suédois, plus précisément de l'Afrique, de l'Asie et de l'Amérique latine. Le site est administré et financé par Världsbiblioteket [la Bibliothèque mondiale], qui loge à Solidaritetsrörelsens hus [la Maison du mouvement de solidarité] à Stockholm. Son but non-lucratif consiste en une construction progressive d'une base de données conceptualisée pour les lecteurs à la recherche d'inspiration, mais aussi à fournir de l'information aux enseignants et aux maisons d'édition qui aspirent à développer des perspectives mondiales dans leurs activités. Le préambule de Världslitteratur.se fut Macondo.nu dont le contenu et les aspects formels ont été élaborés par plusieurs partenaires, entre autres par la maison d'édition Tranan, mais aussi par les usagers intéressés eux-mêmes.

Le site affiche une carte du monde où le navigateur peut faire des recherches d'auteur et de titre par pays (Lindberg, 2010). Comme ce site a eu un impact considérable sur la transmission en Suède de la littérature extra-européenne (Lindberg, 20I0), notre corpus s'appuiera sur ce dispositif accessible au grand public. Världslitteratur.se fait figurer six femmes écrivains, nées après I 960 et situées en Afrique subsaharienne. Il s'agit de Calixthe Beyala et Léonora Miano au Cameroun, Fatou Diome et Marie NDiaye au Sénégal, Bessora au Gabon et, finalement, Nathacha Appanah à l'île Maurice. Dans l'objectif de dévoiler quels critères prévalent dans l'interprétation des auteurs de notre corpus et de leurs œuvres, nous allons procéder à l'analyse de la critique dans la presse journalistique et dans les revues liées aux traductions en suédois de cette littérature catégorisée comme africaine et sub-saharienne sur le site Världslitteratur.se L'analyse inclut Io textes, publiés entre 2003 et 20I3, tandis que le site en répertorie I I selon le tableau ci-dessous. Le 


\section{Tableau 1.}

\begin{tabular}{|c|c|c|c|}
\hline Auteur & Titre original & Titre suédois & $\begin{array}{l}\text { Maison } \\
\text { d'édition }\end{array}$ \\
\hline $\begin{array}{l}\text { Calixthe } \\
\text { Beyala }\end{array}$ & $\begin{array}{l}\text { Les Arbres en parlent } \\
\text { encore Albin Michel } \\
(2002) \\
\text { Les Honneurs perdus } \\
\text { Albin Michel (I996) }\end{array}$ & $\begin{array}{l}\text { Ännu talar träden } \\
(2003) \\
\text { Vår förlorande heder } \\
(2004)\end{array}$ & Leopard \\
\hline $\begin{array}{l}\text { Léonora } \\
\text { Miano }\end{array}$ & $\begin{array}{l}\text { Ces âmes chagrines Plon } \\
\text { (20I I) } \\
\text { Contours du jour qui } \\
\text { vient Plon (2006) }\end{array}$ & $\begin{array}{l}\text { Sorgsna själar (2013) } \\
\text { Konturer av den dag } \\
\text { som nalkas (2008) }\end{array}$ & Sekwa \\
\hline $\begin{array}{l}\text { Fatou } \\
\text { Diome }\end{array}$ & $\begin{array}{l}\text { Le ventre de l'Atlantique } \\
\text { Anne Carrière (2003) } \\
\text { Le vieil homme et la } \\
\text { barque } \\
\text { Naïve (nouvelle, 2010) }\end{array}$ & $\begin{array}{l}\text { Atlantens mage (2010) } \\
\text { Den gamle och baiten } \\
\text { (20II) }\end{array}$ & $\begin{array}{l}\text { Karavan } \\
\text { (revue) }\end{array}$ \\
\hline $\begin{array}{l}\text { Marie } \\
\text { NDiaye }\end{array}$ & $\begin{array}{l}\text { Mon cour à l'étroit } \\
\text { Gallimard (2007) } \\
\text { Trois femmes puissantes } \\
\text { Gallimard (2009) } \\
\text { Ladivine } \\
\text { Gallimard (2013) }\end{array}$ & $\begin{array}{l}\text { Mitt instängda hjärta } \\
\text { (20I2) } \\
\text { Tre starka kvinnor } \\
\text { (20I0) } \\
\text { Ladivine } \\
\text { (septembre 20I4) }\end{array}$ & $\begin{array}{l}\text { Natur } \\
\text { och } \\
\text { Kultur }\end{array}$ \\
\hline Bessora & $\begin{array}{l}\text { Petroleum } \\
\text { Denoël (2004) }\end{array}$ & Petroleum (20I2) & Tranan \\
\hline $\begin{array}{l}\text { Natacha } \\
\text { Appanah }\end{array}$ & $\begin{array}{l}\text { Le Dernier frère } \\
\text { Éditions L'Olivier (2007) }\end{array}$ & $\begin{array}{l}\text { Den siste brodern } \\
\text { (20IO) }\end{array}$ & $\begin{array}{l}\text { Elisabeth } \\
\text { Grate }\end{array}$ \\
\hline
\end{tabular}

roman Ladivine par Marie NDiaye n'a pas été pris en compte lors de la réalisation de l'étude, puisqu'il n'était pas encore publié en suédois. Toutefois, son affichage précoce sur le site signale que les professionnels du livre se servent activement de Världslitteratur.se pour diffuser de l'information.

En comparant les pays scandinaves, il s'avère que le marché du livre suédois est unique, permettant la viabilité d'un certain nombre de petites maisons d'édition spécialisées (Lindberg, 2010 : I75). Cet atout est renforcé par le prix Nobel qui incite à la traduction en suédois dans le but d'attirer l'attention, d'avoir une chance d'être nominé et 
par la suite mondialement diffusés. Par ailleurs, une comparaison avec les Pays-Bas, c'est-à-dire un petit pays en Europe comparable avec la Suède, indique que la Suède accueille plutôt bien la littérature francophone. En parcourant les catalogues des bibliothèques nationales des pays respectifs, il s'avère que le même nombre de titres des auteures Beyala, Diome et NDiaye existent en traduction suédoise et néerlandaise, tandis que Bessora est introuvable en néerlandais. Si deux titres d'Appanah sont traduits en néerlandais, un seul de ses romans est accessible en suédois. Finalement, trois titres de Miano sont traduits en suédois contre un seul en néerlandais. La Suède signale ainsi une légère avance sur les Pays-Bas, une tendance qui pourrait être retracée à l'an 2005 environ, où l'on constate des activités éditoriales soulignant un intérêt croissant dans le domaine de la world literature. Il n'en reste pas moins que la littérature sub-saharienne de langue française traduite en suédois constitue une toute petite part de l'ensemble de la littérature africaine en traduction suédoise, à savoir une vingtaine d'auteurs. Même en incluant les pays de l'Afrique du Nord, tels que l'Algérie, la Tunisie et le Maroc, qui affichent ensemble environ 30 auteurs francophones sur la carte du site Världslitteratur.se, le taux de représentativité reste modeste par rapport à la littérature traduite des pays sub-sahariens anglophones. En effet, le Nigéria et l'Afrique du Sud recensent à eux seuls 80 auteurs sur le site en question.

\section{Les consécrations internationales en cours}

NDiaye se différencie des autres auteures, car elle est la seule à avoir reçu le prix Goncourt, c'est-à-dire l'équivalent français du prix Pulitzer en Angleterre, incarnant la reconnaissance littéraire la plus haute à une échelle nationale (English, 2005 : I7 et Määttä, 2010). De cette manière, NDiaye a pu bonifier son " capital littéraire » (Casanova, I999 : 28) et asseoir sa crédibilité dans la perspective de faire son entrée dans la littérature consacrée. Beyala se rapproche de NDiaye, car elle a également reçu un prix prestigieux, à savoir celui de l'Académie française ${ }^{4}$. Leur consécration en cours se manifeste par l'intégration de leurs biographies dans la publication Dictionnaire littéraire de femmes de langue française : de Marie de France à Marie NDiaye (1996)5. Or, les prix littéraires, tels que Goncourt et Pulitzer, ne sont pas seulement une manière de consolider une littérature nationale, mais incitent également à la traduction et à la mondialisation des auteurs ainsi célébrés (Roberts, 20II). Cette avance sur le marché de NDiaye et de Beyala par 
rapport aux autres auteurs du corpus est indiquée, dans le premier cas par des traductions finnoises de NDiaye, dans le deuxième cas par les chiffres de vente en Suède (Lindberg, 2010 : I73).

La littérature est un moyen efficace pour représenter une identité nationale et pour promouvoir celle-ci dans le monde (Casanova, I999: I 52 ). Toutefois, Roberts montre la confusion dans le sillon de cette pratique de nomination, au moment où les lauréats se caractérisent par leur cosmopolitisme et leurs origines multiples ou seulement étrangères ${ }^{6}$. À cet égard, il est nécessaire de rappeler que NDiaye est le seul auteur du corpus à être née en France. Son nom et sa couleur de peau révèlent certainement des origines qui ne la rattachent pas au premier abord à cette patrie, mais ne l'empêchent pas non plus d'être entièrement de culture et de langue française. Les œuvres du corpus et les maisons d'édition qui les diffusent signalent cette confusion entre " monde » et " nation », car, d'une part, les labels Tranan et Leopard représentent la transmission des littératures d'autres continents de la sphère euro-américaine, d'autre part, Sekwa et Elisabeth Grate affichent un profil francophile, visant à diffuser la littérature francophone et contemporaine, telle qu'elle est présentée par Dominique Viart et Bruno Vercier dans La littérature française au présent (2008).

Ce n'est pas nouveau que la littérature soit interprétée et employée dans un but précis. Par conséquent, les commentaires suédois des œuvres de notre corpus nous aideront à mieux " comprendre le rôle de la critique comme créatrice de valeur littéraire " (Casanova, I999: 36), plus précisément les valeurs portées à la littérature francophone et française.

\section{La critique s'exprime}

Les comptes rendus dans la presse et les reportages sont principalement réunis au moyen des bases de données Media Retriever et Artikelsök, qui recoupent la plupart des quotidiens et revues en Suède. Excepté la presse journalière, nous avons inclus certaines revues littéraires et culturelles pertinentes pour la diffusion de la littérature-monde actuelle, notamment, Karavan, Ord \& Bild, Cora et Vi. Sans prétendre à l'exhaustivité, le résultat est assez révélateur : Calixthe Beyala ${ }^{7}-30$ postes ; Marie Ndiaye - I4 postes ; Léonora Miano - I 5 postes ; Natacha Appanah -6 postes ; Fatou Diome -4 postes ; Bessora -4 postes.

Beyala est sans conteste l'auteure la plus médiatisée du corpus. NDiaye et Miano ont également attiré l'attention de la critique, mais à 
un bien moindre degré. Toutefois, il est intéressant de noter que ces dernières ont fait l'objet de plusieurs reportages assez intimes, qui portent plutôt sur leur personnage d'écrivain que sur leurs œuvres. Il en est de même pour Diome, à qui Karavan a consacré cinq pages pour décrire ce que la journaliste Karin Elfving interprète comme " typique pour la jeune génération d'écrivains africains »[nous traduisons] ${ }^{8}$, à savoir, ayant une origine ex-coloniale et une identité et une vie entre l'Europe et l'Afrique. Ce profil correspond à Karavan qui se définit comme " une revue littéraire en voyage entre les cultures " [nous traduisons]. L'aspect du cosmopolitisme est également mis en avant dans les reportages sur NDiaye dans Helsingborgs Dagblad et Vi läser (Josefsson, 20 I 2 et Dehlén, 20II), dans lesquels est évoquée "l'affaire NDiaye " et son refus de fidélité à la nation française, tout en soulignant un auteur qui n'a pas besoin de patrie pour écrire9. Ce même profil revient dans les entretiens avec Miano où l'écrivain nomme une esthétique et une attitude entre deux continents "l'âme afropéenne » (Börtz, 2008 et Irenius, 2008). Beyala, pour sa part, est connue depuis longtemps pour sa manière d'employer ses attributs culturels des deux continents, aussi bien pour sa littérature que pour se faire écouter dans la société (Lindberg, 2010).

Compte tenu des approches de ces auteures, il faudrait céder aux évidences que Mads Rosendhal Thomsen dissémine dans ses recherches, à savoir la puissante influence de la littérature migrante sur la littérature internationale, une tendance qui semble gouverner l'esprit de la critique suédoise. Selon sa définition, il s'agit d'une littérature par des écrivains dont les œuvres "not really belong to a specific national literature, or at least they have often been so treated » (Rosendahl Thomsen, 2008 : 6I). En d'autres termes, nous avons à faire à une construction hybride de l'identité où l'ancrage dans une géographie spécifique est annulé au profit du nomadisme, souvent urbain (Rosendahl Thomsen, 20I3). Nous observons cette tendance le plus nettement dans l'œuvre de Beyala, qui oscille entre l'occident et l'Afrique subsaharienne, ainsi que chez Diome, qui concilie la géographie et la culture avant et après le voyage vers l'Europe. Cette géographie hybride et globalisante forme également la matière des œuvres de Miano, Bessora et Appanah. Selon Rosendahl Thomsen, cette littérature migrante doit s'évertuer à maintenir une étrangeté limitée en tissant des liens avec la culture occidentale. Si le texte présente uniquement des référents étrangers et locaux, sans représentations liées à une réalité familière, voire euro-américaine, les lecteurs occidentaux sont moins incités à le lire et à le recevoir 
(ibid.). Or, dans l'œuvre de NDiaye, cette oscillation entre terres locales et globales est réduite au seul roman Trois femmes puissantes dont deux des trois histoires se passent en Afrique. Ainsi, il est quelque peu étonnant de voir certains chercheurs et critiques associer l'auteure à, par exemple, Alain Mabanckou, Maryse Condé et Anna Moï dont les origines sont prononcées dans l'ensemble de leurs œuvres respectives (Migraine-George, 2013 : xv).

Dans la critique suédoise émergent des tendances contradictoires en cherchant à cerner cette littérature importée. En s'appuyant de prime abord sur les comptes rendus publiés dans Helsingborgs Dagblad, le seul quotidien qui s'est montré attentif à chaque écrivain du corpus, il s'avère que l'exotisme, l'authenticité, le colonialisme et la world literature sont les mots d'ordre ${ }^{10}$. Dans ce contexte, Diome est le seul des écrivains à être critiquée pour sa création romanesque médiocre. Comme dans le compte rendu de Ces âmes chagrines de Miano, la critique émet des réserves entre autres concernant son exotisme trop prononcé. Dans la critique d'Appanah cet exotisme est transformé en une authenticité approuvée, faisant appel à une branche des études postcoloniales qui souligne le local, les nouvelles identités nationales et des voix authentiques (Rosendahl Thomsen, 20I3). Beyala et Bessora, pour leur part, sont louées pour leur perspective "mondialisante » lorsqu'elles s'évertuent de représenter le colonialisme et ses conséquences. La comparaison entre le langage rocambolesque et caustique de Bessora avec celui de Beyala vient renforcer le lien entre ces auteures. La critique exprime ouvertement à l'égard de Beyala qu' "indépendante et confiante elle occupe une place dans le monde et dans la world literature » [nous traduisons $]^{\text {II }}$. Ce même jugement positif se manifeste également dans les propos concernant Bessora et Miano. Cette dernière est en outre associée à un des premiers romanciers européens dont l'œuvre est marquée par le colonialisme et la world literature, à savoir Joseph Conrad.

Il n'en reste pas moins que NDiaye n'y trouve pas sa place, car, toujours dans Helsingborgs Dagblad, elle est l'unique écrivain clairement associé à un canon littéraire occidental, notamment par les ressemblances avec l'œuvre de Franz Kafka, à propos de son roman Mon cour à l'étroit. De plus, dans Karavan, on souligne que NDiaye est dissociée de toute théorie postcoloniale prônant « le sujet nomade où l'existence en exil est une porte vers des possibilités transnationales et transculturelles " [c'est nous qui traduisons] ${ }^{12}$. Par ailleurs, dans Expressen, Per Wirtén déplore le fait que le roman Trois femmes puissantes ait joui d'aussi peu d'attention en Suède, malgré le prix Goncourt. Peut-être ce 
désintérêt témoigne-t-il de l'image de la littérature francophone comme minoritaire, à l'écart des systèmes de consécration. Il se peut aussi que la réconciliation du francophone et du français innée de l'œuvre de NDiaye est inconcevable pour les critiques qui ne sauraient pas la commenter. En tout état de cause, Ingrid Elam ironise dans Dagens Nyheter autour de l'accueil parfois réducteur en Suède de NDiaye, en écrivant que c'est grâce au thème africain au Salon du Livre à Göteborg que le dernier roman de l'auteure sera traduit en suédois ${ }^{13}$. Elle continue en expliquant que " c'est une ironie historique : pour un écrivain français comme Marie NDiaye, le chemin vers le succès passe par le monde postcolonial qu'elle décrit et dont elle fait partie » [nous traduisons] ${ }^{\mathrm{I} 4}$. Ces propos signalent un certain effort de la part des critiques à vouloir rallier NDiaye à la tradition littéraire française. Kullberg voit même en NDiaye "le sauveur du roman moderne ${ }^{\mathrm{I}}{ }^{5}$ et l'héritière de Flaubert. La reconnaissance de NDiaye comme un écrivain français fait contraste avec la critique dans Ord \& Bild, la revue culturelle la plus ancienne et une des plus prestigieuses en Suède, où Mikaela Lundahl, auteur de Vad är en neger? Negritude, essentialism, strategi (2005), suggère que NDiaye s'inscrit avec Trois femmes puissantes dans une tradition africaine et qu'elle offre une image stéréotypée de la femme africaine souffrante.

\section{Conclusion}

La réception en Suède de ces six écrivains bien ancrés dans le monde d'édition en France tend à observer en premier l'écriture aux empreintes postcoloniales, tout en étant sensible à l'expression des liens et des mouvements entre plusieurs lieux géographiques et culturels. Ce type d'interprétation domine l'évaluation des critiques, qui, par moments, voient de la littérature africaine et postcoloniale là où on a plutôt à faire à une littérature "transculturelle " où plusieurs cultures ont voix au chapitre, tout en laissant à la culture et à la langue de l'écriture une place dominante ${ }^{16}$. Il n'est pas sans intérêt que NDiaye figure dans le corpus réuni sur le site Världslitteratur.se en tant que représentante du Sénégal. Les critiques avérés font montre d'un modeste scepticisme vis-à-vis de cette labellisation francophone et essaient d'y remédier en comparant son œuvre avec la littérature française canonisée. En effet, à travers les auteurs auxquels les critiques font référence pour décrire les œuvres de notre corpus, il est possible d'observer différentes formes de reconnaissance. Tantôt la critique fait valoir le canon européen ou français, tan- 
tôt le canon world ou africain sub-saharien. Cette pratique ne doit pas être interprétée comme post- ou néocoloniale. Il s'agit plutôt du besoin de comprendre une littérature par rapport à une norme, afin de saisir son sens et son esthétique. Ces six auteures intègrent clairement dans leurs œuvres un regard à la fois africain et européen, créant de cette manière une forme de littérature migrante diffusable dans le monde. Ce contexte ouvre de nouvelles perspectives pour étudier la littérature francophone, qui, de toute évidence, n'est pas une littérature marginale, comme le suggèrent les auteurs dans Pour une littérature monde (2007), mais plutôt une force pour freiner « le déclin de la littérature française » dans le monde (Le Bris \& Rouaud, 2007 et Taylor, 20II).

\section{Notes}

I. Pour cette étude nous employons le terme world literature pour désigner la littérature qui circule dans le monde et dont la réception est notable. Voir Damrosch (2003).

2. Voir Helgesson, (I999, 2004 \& 2008) et Lindberg-Wada \& Helgesson (2006).

3. Voir Gunder (20II), Engdahl (2008) et D’haen, Domínguez, \& Rosendahl Thomsen (2013).

4. Cette célébration de son roman Les Honneurs perdus (I996) a été suivie d'une contestation ferme, surtout de la part du critique Pierre Assouline accusant Beyala de plagiat (Hitchcott, 2006).

5. Les deux auteures ont eu autant d'espaces dans le dictionnaire, à savoir trois pages d'information (Makward \& Cottenet-Hage, I996).

6. Anthony Appiah exemplifie le dilemme de vouloir définir une identité nationale tout en reconnaissant ses origines pluriculturelles (Appiah, 2007 : I I 8 sq.).

7. Pour les articles sur Beyala, nous nous appuyons sur une recherche effectuée en 2010 (Lindberg, 2010).

8. « Hon kan betraktas som typisk för den yngre generationen afrikanska författare » (Elfving, 20II).

9. "L'affaire NDiaye " est rapportée dans la thèse de doctorat de Thérèse Migraine-George, qui reprend l'expression « cosmopolitanisme partiel » de K. A. Appiah pour définir une littérature qui exprime la jonction de plusieurs allégeances : culturelles, locales, nationales, communautaires, tout en faisant 
montre d'une "loyauté vis-à-vis de toute l'humanité »[nous traduisons]. (Appiah, 2007 : xvi sq) et (Migraine-George, 20I3: x).

Io. Il est probablement significatif que Helsingborg est le fief de la maison d'édition Sekwa.

I I. « [...]självständigt och självsäkert tar plats i världen och världslitteraturen ». Helsingborgs Dagblad (2003) : "Afrika sett med kvinnliga ögon ».

I2. «[...] det nomadiska subjektet där tillvaron i exil ses som en dörr mot transnationella och transkulturella möjligheter ». (Kullberg, 20I3)

I3. Elam fait allusion au roman Mon cour à l'étroit que tous les critiques ne semblent pas avoir intégré comme une publication antérieure à Trois femmes puissantes.

I4. " En historisk ironi, vägen till uppmärksamhet för en fransk författare som Marie NDiaye går via den postkoloniala värld hon både beskriver och är en del av»(Elam, 2010).

I 5. « den moderna romanens räddare » (Kullberg, 20I3).

I6. Chirila, I. (20I2), La République réinventée: littératures transculturelles dans la France contemporaine. Thèse de doctorat, Duke University.

\section{Bibliographie}

Appiah, K. A. (2007) [2006], Cosmopolitanism. Ethics in a World of Strangers. London : W. W. Norton.

Börtz, T. (2008), " Miano står för sin bild av Afrika ». Göteborgs-Posten, 3 décembre.

Casanova, P. (I999), La République mondiale des Lettres. Paris : Seuil.

Coly, A. A. (2010), The Pull of Postcolonial Nationhood. Gender and Migration in Francophone African Literatures. United Kingdom : Lexington Books.

Coste, C. (2010), «La France est-elle un pays francophone?». Recherches \& Travaux $76:$ 9I-I07.

Damrosch, D. (2003), What is World Literature?. Princeton : Princeton University Press.

Dehlén, J. (20I I), « Presidentens fiende ». Vi läser 5 : 55-59.

D’haen, T., Domínguez, C. \& Rosendahl Thomsen, M. (éd.) (2013), World Literature. A Reader. New York : Routledge.

Elam, I. (20I0), "Själens smärta ger djupa sår ». Dagens Nyheter, 3 juillet. 
Elfving, K. (20II), " "Jag kan skriva allt jag vill på franska” ". Karavan 3 : I 6-20.

Engdahl, H. (2008), "Canonization and World Literature », in Simonsen, K.M. \& Stougaard-Nielsen (éd.), World Literature, World Culture. History, Theory, Analysis. Aarhus : Aarhus UP : I95-2I4.

English, J. F. (2005), The Economy of Prestige. Prizes, Awards and the Circulation of Cultural Value. Cambridge, Mass. : Harvard University Press.

Fanon, F. (2002) [I96I], Les Damnés de la terre. Paris : Découverte/Poche.

Gunder, A. (20II), "In the Wake of a Nobel Prize. On Modern Icelandic Literature in Swedish I940-1969 ", in Broomans, P. \& Jiresch, E. (éd.), The Invasion of Books in Peripheral Literary Fields. Transmitting Preferences and Images in Media, Networks and Translation. Groningen : Barkhuis : I05-I I6.

Helgesson, S. (2007), "Afrikanska kvinnor stiger in i centrum ». Dagens Nybeter, 20 juin.

- (1999), Sports of Culture. Writing the Resistant Subject in South Africa (readings of Ndebele, Gordimer, Coetzee). Thèse de doctorat, Uppsala : Uppsala University.

- (2004), Writing in Crisis: Ethics and History in Gordimer, Ndebele and Coetzee. Pietermaritzburg : University of Kwazulu : Natal Press.

- (2008), Transnationalism in Southern African Literature : Modernists, Realists, and the Inequality of Print Culture. London : Routledge.

Helsingborgs Dagblad (2003), "Afrika sett med kvinnliga ögon », I9 août.

Hitchcott, N. (2006), "Calixthe Beyala. Prizes, Plagiarism, and 'Authentity' ". Research in African Literature 37 (vol.I) : I00-109.

Irenius, L. (2008), « En afropeisk själ ». Dagens Nybeter, I 8 octobre.

Josefsson, E. (2012), " Med skarp blick på dåligt samvete ». Helsingborgs Dagblad, I9 juin.

Kullberg, C. (2013), « Genialiskt om hemlösa nomaders utsatthet ». Karavan I : 79-80.

Le Bris, M. \& Rouaud, J. (2007), Pour une littérature-monde. Paris : Gallimard.

Lindberg-Wada, G. \& Helgesson, S. (éd.) (2006), Literary History : Towards a Global Perspective (Vol. 4). Literary Interactions in the Modern World. 2, Berlin, W. de Gruyter.

Lindberg, Y. (2010), "Calixthe Beyala chez les Scandinaves ». Présences francophones $75:$ I $67-\mathrm{I} 86$. 
Lundahl, M. (2005), Vad är en neger? Negritude, essentialism, strategi. Göteborg : Glänta.

Makward, C. P. \& Cottenet-Hage, M. (éd.) (I996), Dictionnaire littéraire des femmes de langue française. De Marie de France à Marie Ndiaye. Paris : Karthala.

Migraine-George, T. (2013), From Francophonie to World Literature in French: Ethics, Poetics \& Politics. Thèse de doctorat, Lincoln : University of Nebraska Press.

Määttä, J. (2010), " Pengar, prestige, publicitet. Litterära Priser och utmärkelser i Sverige I786-2009 ". Samlaren I 3 I : 232-329.

Roberts, G. (20II), Prizing Literature. The Celebration and Circulation of National Culture. Toronto University of Toronto Press.

Rosendahl Thomsen, M. (2008), Mapping World Literature. International Canonization and Transnational Literatures. London, New York : Continuum Literary Studies.

Rosendahl Thomsen, M. (2013), "Strangeness and World literature ". CLCWeb : Comparative Literature and Culture I5 (vol. 5) : article I8. Consulté le 28 septembre 20I 5, http://docs.lib.purdue.edu/cgi/viewcontent. cgi? article $=235$ I \& context $=$ clcweb

Taylor, J. (20II), "Le déclin de la littérature française ? ", in Viart, D. \& Demanze, L. (éd.), Fins de la littérature. Esthétiques et discours de la fin, tome I. Paris : Armand Colin/Recherches : I6I-I70.

Viart, D. \& Vercier, B. (2008) [2005], La littérature française au présent. Paris : Bordas. 